\title{
Evaluation of Accuracy between Habit History and Incidence of Oral Squamous Cell Carcinoma
}

\section{Snega Thamilselvan ${ }^{1}$, Abilasha $\mathbf{R}^{2}$, Pratibha Ramani ${ }^{3}$, Gheena $\mathbf{S}^{4}$, Hannah $\mathbf{R}^{5}$}

Section: Healthcare Sci. Journal Impact Factor: 6.1 (2018) ICV: 90.90 (2018)

(c) (7) (8)

Copyright@IJCRR

\begin{abstract}
'Department of Oral and Maxillofacial Pathology, Saveetha Dental College and Hospitals, Saveetha Institute of Medical and Technical Sciences, Chennai, Tamil Nadu, India; 'Reader, Department of Oral and Maxillofacial Pathology, Saveetha Dental College and Hospitals, Saveetha

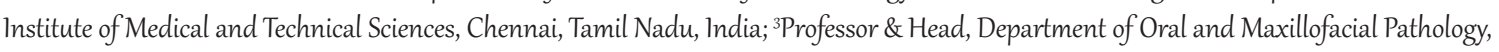
Saveetha Dental College and Hospitals, Saveetha Institute of Medical and Technical Sciences, Chennai, Tamil Nadu, India; ${ }^{4}$ Reader, Department of Oral and Maxillofacial Pathology, Saveetha Dental College and Hospitals, Saveetha Institute of Medical and Technical Sciences, Chennai, Tamil Nadu, India; ${ }^{5}$ Senior Lecturer, Department of Oral and Maxillofacial Pathology, Saveetha Dental College and Hospitals, Saveetha Institute of Medical and Technical Sciences, Chennai, Tamil Nadu, India.
\end{abstract}

\section{ABSTRACT}

Introduction: Oral squamous cell carcinoma (OSCC) is one of the most common cancers that affect the human population worldwide. The global incidence rate of OSCC is $47 \%$ which shows a recurrence of $30 \%$.

Objective: The aim of this study is to analyze the accuracy between risk factors and the incidence of OSCC.

Methods: This retrospective study includes the subjects reported to the Department of Oral Pathology and Microbiology in Saveetha Dental College between 2019- 2020. Data on age, gender, lesion site, habit profile, diagnosis, and treatment were recorded and subjected to Pearson's Chi-square analysis for correlation between habits and other variables.

Results: A total of $n=77$ cases were included. Male: Female ratio is 3.5:1 with maximum cases in the 5 th and 6 th decades of life. Totally $63.3 \%$ reported OSCC without habits and $36.7 \%$ reported OSCC with habits pan/betel quid chewing was the most frequently reported habit and buccal mucosa was the common site.

Conclusion: Our study showed that the incidence of OSCC is more among the male population in the 4th - 6th decade with at least one predisposing oral habit and identified buccal mucosa to be the most commonly involved site. This study reflects the association of oral habits with the incidence and severity of the disease which quantifies the need for awareness for habit cessation among the public.

Key Words: Accuracy, Habits, Risk factor, Incidence, OSCC, Institutional level, Smoking, Tobacco

\section{INTRODUCTION}

Worldwide oral cancer accounts for $2 \%$ of all cancer cases. ${ }^{1}$ Oral squamous cell carcinoma (OSCC) is the most common oral malignant epithelial neoplasm, respectively up to $80 \%$ to $90 \%$ of all oral malignant neoplasms of the oral cavity. ${ }^{2}$ The incidence of OSCC accounts for about $47 \%$ globally with its local recurrence rate being $30 \%$. The chief risk factor for the OSCC development is stated as tobacco and alcohol usage which is a potential carcinogen. The most common type of tobacco prevalent in India is betel quid chewing. The risk increases dramatically when alcohol use exceeds $6 \mathrm{oz}$ of distilled liquor (or) $15 \mathrm{oz}$ of wine (or) $36 \mathrm{oz}$ of beer (or) more than 2 packs of cigar per day. Bradley A Schiff evaluated that incidence of oral cancer to be six times more likely to devel- op in alcohol/tobacco users than non-smokers/non-drinkers. The combination of tobacco and alcohol users has fifteenfold risk to develop oral cancer. The chronic exposure to these potential carcinogens activates the genetic damage that causes mutations or amplification of the oncogenes which furthers lead to cell proliferation or cell death. ${ }^{3-5}$ There are also non - habit etiological factors such as impaired ability to repair DNA damage by mutagens ${ }^{6}$, impaired ability to metabolize carcinogens, deficiencies of vitamin A / E / C and also immune defects. ${ }^{3}$ The estimated death due to oral cancer every year is $43 \%$ due to tobacco use, alcohol consumption, unhealthy diets, inactive lifestyle, and severe infections. ${ }^{7}$

There are also a few similar retrospective studies that are performed at an institutional level. Ranganathan et al., 2015 have done retrospective analysis on OSCC cases (151 OSCC cases)

\section{Corresponding Author:}

Dr. Abilasha R, Reader, Department of Oral and Maxillofacial Pathology, Saveetha Dental College and Hospitals, Saveetha Institute of Medical and Technical Sciences, Chennai, Tamil Nadu, India; Email: abilasha@saveetha.com

ISSN: 2231-2196 (Print)

Received: 16.09 .2020
ISSN: 0975-5241 (Online)

Revised: 19.10 .2020
Accepted: 14.11 .2020
Published: 26.12 .2020 
with or without predisposing habits which is a 13.7 year period study. He determined that $74.8 \%$ of the affected populations have at least one predisposing habit. ${ }^{8}$ Salian et al., in 2016 have done a retrospective cross-sectional study with 61 cases of OSCC in which $96.7 \%$ of the study population were analyzed to have a tobacco-chewing habit which is a 5 year period study at the institutional level. ${ }^{9}$ Tandon et al., in 2017 performed a retrospective study at the institutional level - A 10 year period study on 1020 SCC cases and analyzed the habitual profile of the OSCC patients. ${ }^{10}$ This study prevalence and predictors of OSCC with diverse habitual practices. ${ }^{11}$

OSCC is a disease that severely affects the basic human functions but still, it is a largely preventable disease through lifestyle choices that reduce one's risk for disease. ${ }^{12}$ Relatively high tobacco has led to an increase in incidence because of the unawareness of the initial cancerous lesion, irregular follow-ups, and limited access to cancer care. ${ }^{10,13}$ This research is done to quantify the need for related patient awareness and to make the necessary clinical interventions and it also helps to access the recurrence rate among patients at the institutional level. This study is done to analyze the accuracy of the oral habitual history with the incidence of OSCC in patients at an institutional level.

\section{MATERIALS AND METHODS}

This is a retrospective study performed with clinically and pathologically diagnosed patients of OSCC referred to the Department of Oral Pathology and Microbiology in Saveetha Dental College and Hospitals during 2019 - 2020. The study was conducted after getting approval from the ethical committee of Saveetha Dental College and Hospitals. The data was analyzed and collected among 86000 patient records from the management software of the institution. The demographic details - age, gender, habit profile, lesion site, investigation, diagnosis, and treatment history were retrieved and tabulated. The age of the patients was categorized as $<50$ years and $>50$ years. The oral habits were further classified based on the form of the tobacco, this included smoking tobacco/smokeless tobacco, and also those who have multiple habits were categorized accordingly. The site of the lesion included buccal mucosa, lateral border of the tongue, upper/ lower jaw, gingivobuccal sulcus, and retromolar region. The collected data are clinicopathologically confirmed cases of OSCC which were cross verified with the photographs to minimize the sampling bias. The patients who weren't reviewed after the initial/provisional diagnosis were excluded from the study.

\section{Statistical Analysis}

All the collected data were entered and statistically analyzed using SPSS software version 20.0, IBM. The frequency tables were done for all the parameters. A Chi-square test was performed to statistically identify the correlation between the habit profile and diagnosis. Also, for finding the association of cross-tabulation. A p-value less than or equal to 0.05 was considered statistically significant.

\section{RESULTS AND DISCUSSION}

A total of $n=77$ clinically and pathologically diagnosed OSCC cases were included in the study for analyzing the accuracy between the risk factors and incidence of OSCC.

The habit profiles of $n=77$ cases were assessed and $63.64 \%$ of patients didn't have any propensities and $36.36 \%$ of patients had at least one inclining habit (tobacco in any form) (Graph 1).

The age distribution of $n=77$ cases was found to be $46.75 \%$ of patients were $<50$ years of age and $53.25 \%$ were $>50$ years of age (Graph 2).

The gender variations in our study showed a predominance of the male population with $77.92 \%$ and $22.08 \%$ of the patients were female (Graph 3).

The anatomical site involvement of $\mathrm{n}=77$ OSCC patients was found to be, $44.16 \%$ were buccal mucosa, $19.48 \%$ were the lateral border of the tongue, $22.08 \%$ were upper/lower jaw, $3.90 \%$ were retromolar region and $10.39 \%$ were gingivobuccal sulcus (Graph 4).

The habit profile of $n=77$ patients was $1.30 \%$ had smoking tobacco, $16.88 \%$ had a pan, $5.1 \%$ had gutka, $1.30 \%$ had areca nut, $11.69 \%$ had tobacco in multiple forms and $63.64 \%$ had no habits (Graph 5).

The confirmatory histopathological diagnosis of SCC variants have showed WDSCC (55.8\%), MDSCC (19.5\%), SCC $(18.2 \%)$, PDSCC (5.2\%), and verrucous into SCC $(1.3 \%)$ (Graph 6).

The association between age \& gender with habits; habits with OSCC are further represented in (Graph 7-9). The correlation between the habitual profile and incidence of OSCC has been examined utilizing a chi-square test, which showed a $p$-value $>0.05$, insignificant result $(p=0.885)$. Statistically, the correlation between the parameters couldn't be justified.

The changing trends of habits and the rate of occurrence of OSCC have been described in this literature. ${ }^{14,15}$ Oral cavity cancer is the 8th most frequent cancer among males and the 14th most frequent cancer among females globally with the major risk factor being tobacco and alcohol consumption. ${ }^{16}$ Oral cancer in India is a major public health problem. International agency for research on cancer mentions that cancer of the oral cavity, lungs, esophagus, stomach, cervix, and breast are commonly occurring cancer in the Indian population. ${ }^{9,17}$

A total of $n=77$ cases were included in this study. The age range of the patients in our study was between 32 and 71 
years with a mean age of 57 years. The study results showed that more OSCC cases were seen in the 4th-6th decade of life. This is in accordance with Mathur et al. ${ }^{18}$ who reported that OSCC commonly occurs in the 5th - 6th decade of life. ${ }^{19}$ Oral cancers in young individuals follow a worse aggressive course and are associated with poor prognosis..$^{20,21}$ Our study also had a young patient of 32 years of age.

The male: female ratio in our study was estimated at 3.5:1. The results showed the male population was more affected with OSCC..$^{22,23}$ This is in accordance with the study by Sharma et al. ${ }^{24}$ in 2010, who found that the male predominance is more in the North Indian population of OSCC. Mennalves et al., in $2017^{25}$ found that male populations are more affected by OSCC than female populations. The reason for the male predominance with the incidence of OSCC may be because of the cultural differences and social norms in different sectors, especially in India. In Contrast, Pindborg et al. observed that the concept of reverse smoking is more in Andhra Pradesh that affects more of the female population. ${ }^{26}$

Our study analyzed and found that the most common site of occurrence for OSCC to be buccal mucosa followed by the tongue. ${ }^{22,27}$ This is probably because the majority of the lesion corresponds to the site of maximum exposure to tobacco and other related habits. Rai et al., 2016 in their analysis also found buccal mucosa to be the most common site for OSCC. Tandon et al., in $2016^{10}$ found that $31.47 \%$ incidence of OSCC is seen in the buccal mucosa. Thus our results are in accordance with previous findings.

The most common risk factor found in our study was pan chewing. Our study also found that $63.3 \%$ of cases had no habits which are not in accordance with any previous literature. Ranganathan et al., $2015^{8}$ stated $74.8 \%$ of OSCC cases were determined to have at least one predisposing habit. Salian et al., $2016{ }^{9}$ found $96.7 \%$ cases were analyzed with tobacco chewing habits to develop the lesion. The factor which affected the results is the minimum number of samples. Quid, which is also known as pan, consists of betel leaf, areca nut, tobacco, and lime. ${ }^{28}$ Quid chewing is proved to be an independent risk factor for oral cancer. ${ }^{29}$ The combination of lime and tobacco causes an exothermic reaction which increases the susceptibility of oral mucosa to carcinogens. ${ }^{30}$ This variation of our study can be attributed to the geographic limitation and minimum sample size of our study. Currently, there are various able sources that emphasize the risk of usage of tobacco with the development of cancer, and that created huge awareness among the people. Even though the usage of tobacco has decreased greatly in present times, habit cessation widely around the globe remains a question. The study results showed that pan chewing habit was more among OSCC with habits. Betel quid is known to induce proliferation and differentiation of oral epithelial cells. ${ }^{31,32}$ It has been associated with src family kinases released from betel quid have been associated with cell migration and invasive potential of OSCC indicating a poor prognosis. ${ }^{33,34,35}$, The increased pan habit is because of the easy availability and affordability.

The confirmatory histopathological diagnosis showed different variants of OSCC. The highest number of cases in our study was well-differentiated SCC, followed by moderately differentiated SCC with pan/ betel quid having its more predominance. This is because the patients report to hospitals only when their quality of life is disturbed to a greater level. Though the knowledge of cancer is sufficient, the patient's mental attitude to accept the presence of cancer prohibits them from visiting a hospital at an early stage.

\section{Limitations}

Availability of the samples. Data not specified are not included in the study. It is an institutional level based study. Even though the statistical significance of the study is not in accordance, this research quantifies the need for related patient awareness which is necessary for clinical interventions that help to access the recurrence rate.

\section{CONCLUSION}

The present study estimated the accuracy between the oral habitual practices with the incidence of OSCC. The study results revealed that occurrences of OSCC are more in the male population in the 4th - 6th decade of life and buccal mucosa was the predominant site. There was no habit profile in more than half of the cases which is an unanticipated result of the study. Thus, this study encourages people with no habits as well as to initiate an awareness campaign for people with habits for habit cessation to further prevent the incidence of OSCC at an institutional level. However, Large scale longitudinal studies are required to substantiate the findings obtained.

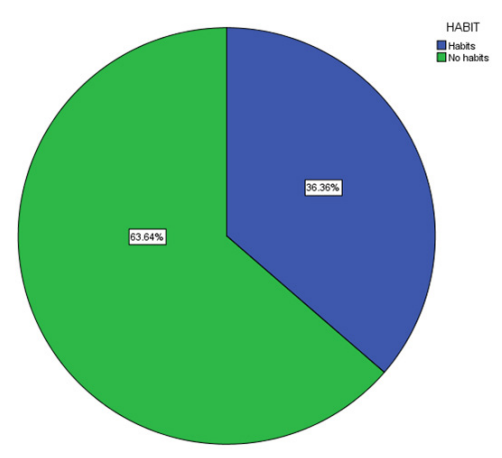

Graph 1: Pie chart depicting the habitual status among the OSCC patients at an institutional level. The OSCC patients with habits are represented in green color with $63.64 \%$ and OSCC patients without habits are represented in blue color with $36.36 \%$. 


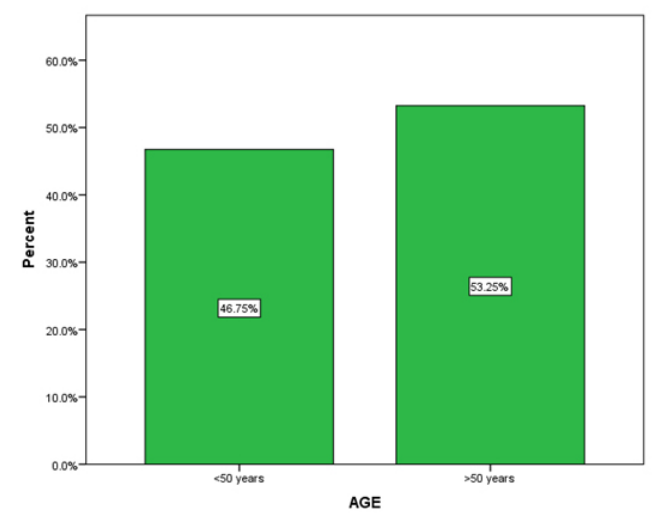

Graph 2: Bar chart represents the frequency distribution of age among OSCC patients. The $\mathrm{X}$-axis denotes the age and Y-axis denotes the percentage of cases. $46.75 \%$ of patients were $<50$ years of age and $53.25 \%$ were $>50$ years of age.

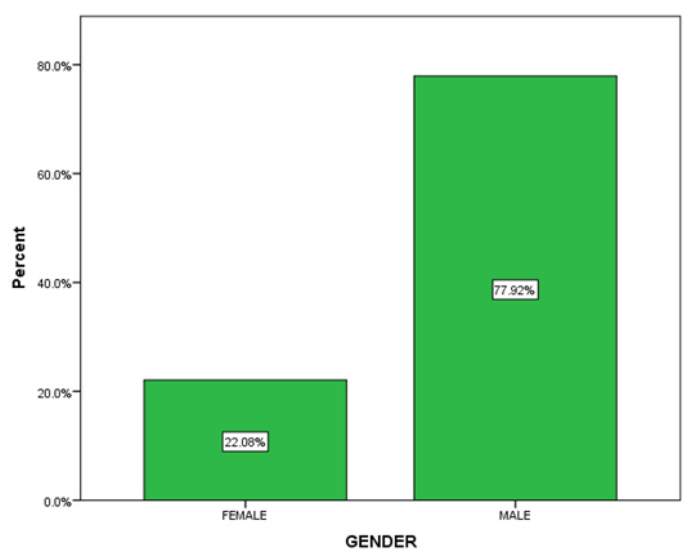

Graph 3: Bar chart shows the frequency distribution of gender among the OSCC patients. The X-axis denotes the gender and $\mathrm{Y}$-axis denotes the percentage of cases. $22.08 \%$ of the patients were female and $77.92 \%$ were male.

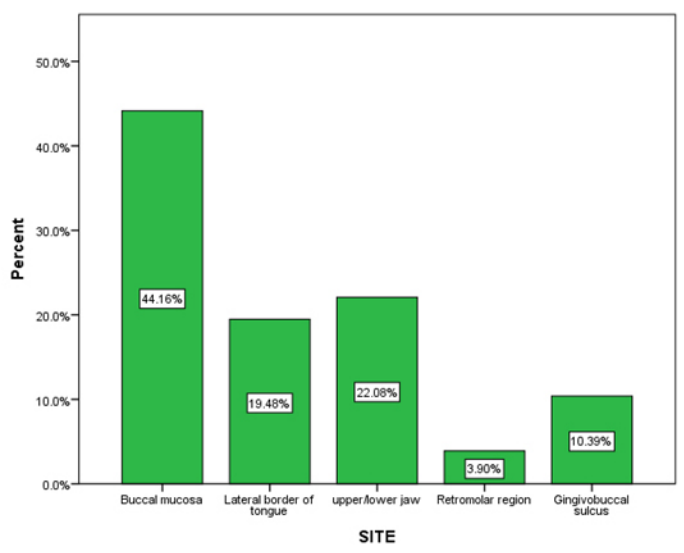

Graph 4: Bar chart depicting the frequency distribution of involved anatomical sites among OSCC patients. The $\mathrm{X}$-axis denotes the site and $\mathrm{Y}$-axis denotes the percentage of cases. The anatomical site of OSCC patients was $44.16 \%$ buccal mucosa, $19.48 \%$ were the lateral border of the tongue, $22.08 \%$ were upper/lower jaw, $3.90 \%$ were retromolar region and $10.39 \%$ were gingivobuccal sulcus.

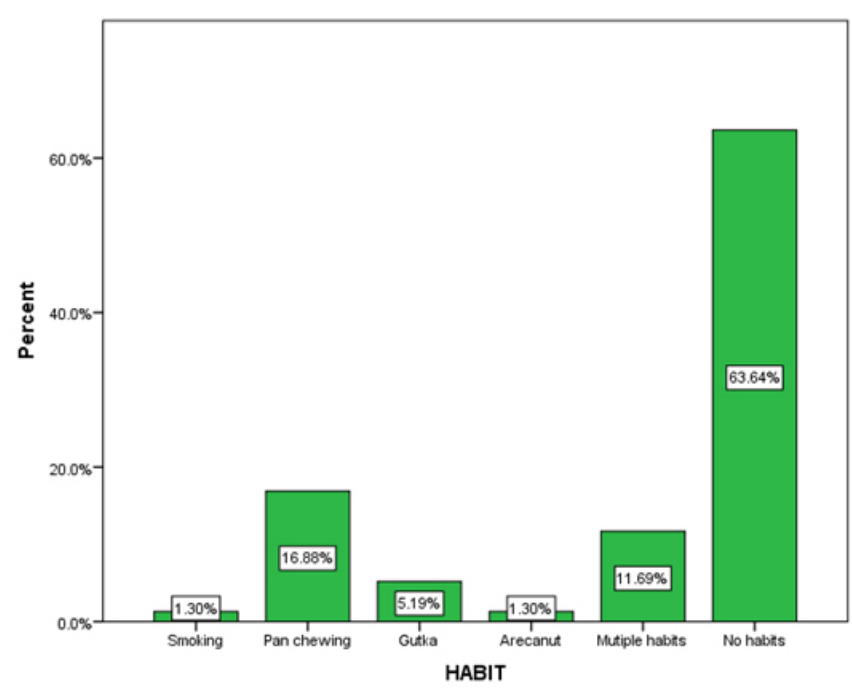

Graph 5: Bar chart depicting the frequency distribution of habits profile among OSCC patients. The $\mathrm{x}$-axis denotes the habit and $Y$-axis denotes the percentage of OSCC cases. The habit profile of OSCC patients was $1.30 \%$ had smoking tobacco, $16.88 \%$ had a pan, $5.1 \%$ had gutka, $1.30 \%$ had areca nut, $11.69 \%$ had tobacco in multiple forms and $63.64 \%$ had no habits.

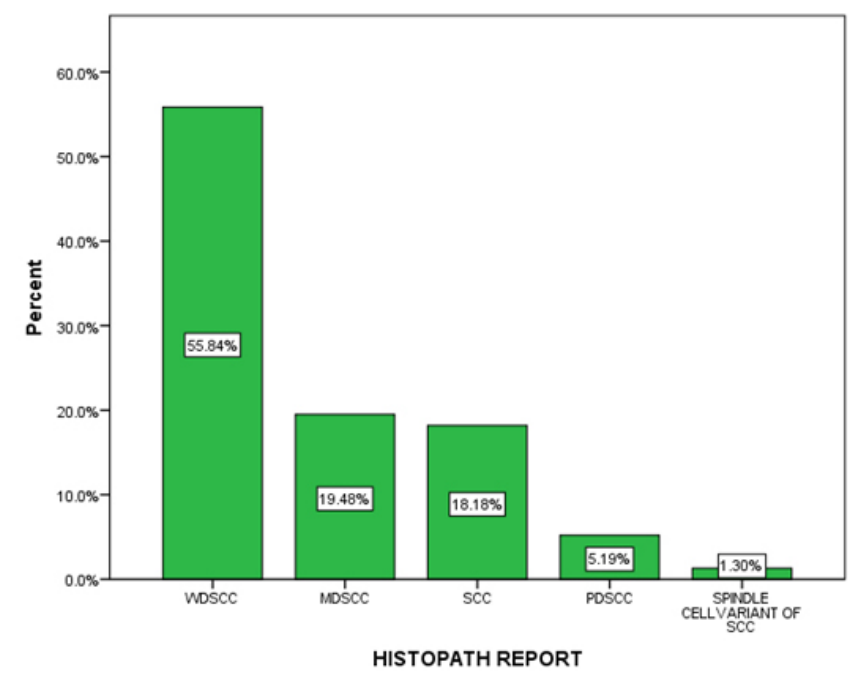

Graph 6: Bar chart depicting the confirmatory histopathological diagnosis of OSCC patients. The X-axis denotes the histological diagnosis and $\mathrm{Y}$-axis denotes the percentage of OSCC cases. The WDSCC was seen in $55.84 \%$, MDSCC has seen in $19.84 \%$, SCC was seen in $18.18 \%$, PDSCC was seen in $5.19 \%$, and spindle cell variant of SCC seen in $1.30 \%$. 


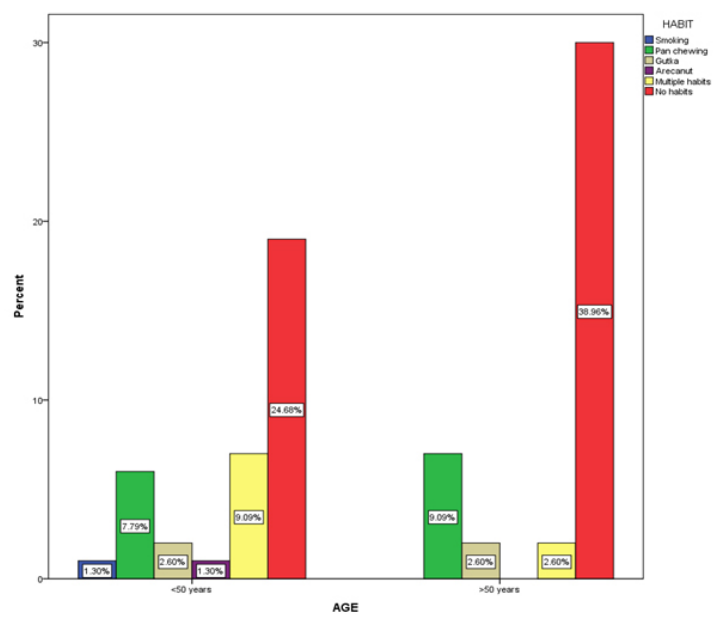

Graph 7: Bar chart depicting the association between the age and the habit profile of OSCC patients. The X-axis denotes age and types of habits and the Y-axis denotes the percentage of habits. The smoking habit is represented in blue color, the pan chewing habit is represented in green color, the habit of gutka is represented in sandal color, the habit of areca nut is represented in purple color, yellow color shows the multiple habits and no habits is represented in red color. In both the age categories, the cases with no habit profile are more followed by the predominance in pan chewing habit. Pearson's chi-square statistical test showed $p$-value $=0.218(p>0.05$ statistically insignificant).

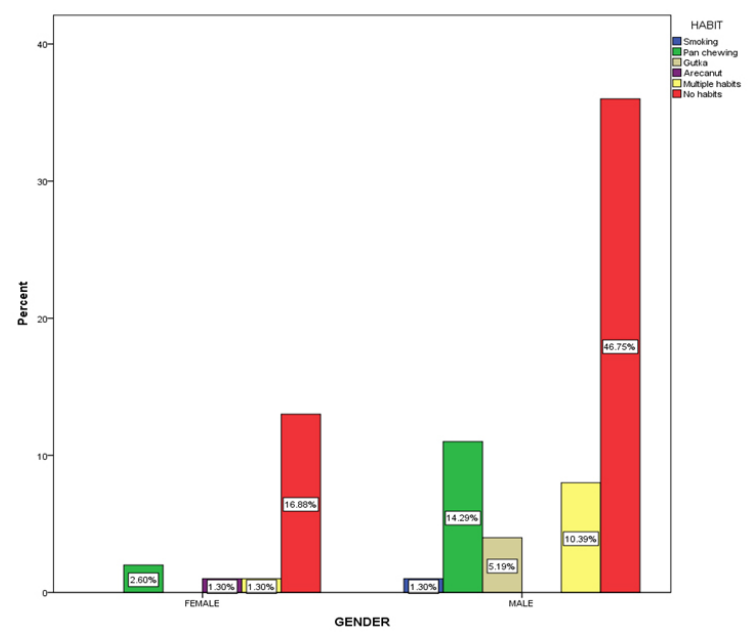

Graph 8: Bar chart depicting the association between Gender and oral Habit profile of OSCC patients. The X-axis denotes Gender and types of habits and the Y-axis denotes the percentage of habits. The smoking habit is represented in blue color, the pan chewing habit is represented in green color, the habit of gutka is represented in sandal color, the habit of areca nut is represented in purple color, yellow color shows the multiple habits and no habits is represented in red color. In both the groups (females and males), the cases with no habit profile are predominant. Pearson's chi-square statistical test showed $p$-value $=0.262$. $(p>0.05$ - statistically insignificant $)$.

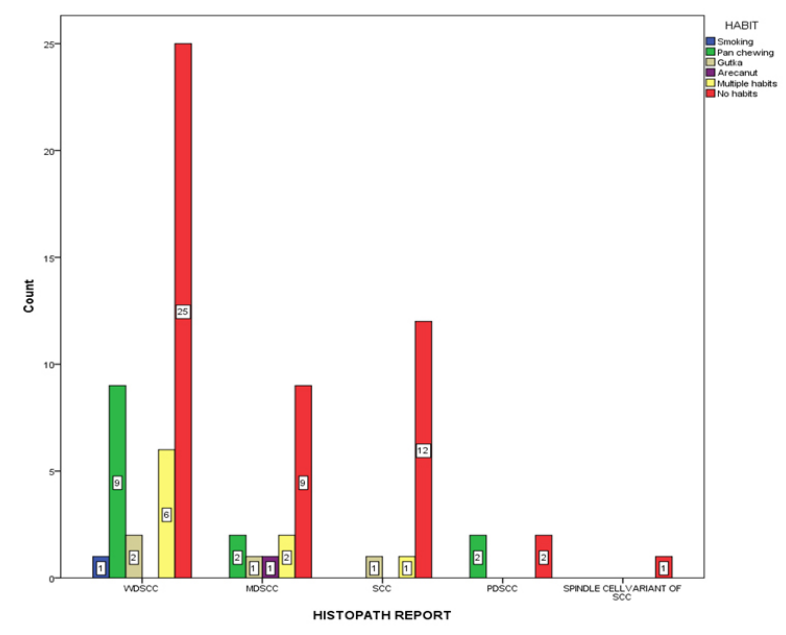

Graph 9: Bar chart depicting the association between habit and confirmatory histopathological diagnosis of OSCC patients. The $\mathrm{X}$-axis denotes the histopathological diagnosis and Y-axis denotes the number of OSCC cases. The smoking habit is represented in blue color, the pan chewing habit is represented in green color, the habit of gutka is represented in sandal color, the habit of areca nut is represented in purple color, yellow color shows the multiple habits, and no habits is represented in red color. The predominance of no habits among all the categories was observed. Pearson's chi-square statistical test showed $p$-value $=0.885(p>0.05$ - statistically not significant).

\section{ACKNOWLEDGMENT}

The authors would like to acknowledge the help and support rendered by the Department of Oral Pathology and Microbiology of Saveetha Dental College and Hospitals and the management for their constant assistance with the research.

\section{Authors' Contributions}

Snega Thamilselvan conceived and carried out experiments, Dr. Abilasha Ramasubramanian and Dr. Pratibha Ramani contributed to the design and analyzed the data. All the authors contributed to the analysis of the results and writing the paper and approved the final manuscript.

\section{Conflict of Interest}

The authors declare that they have no competing interests.

Funding: No funding.

\section{REFERENCES}

1. Ghafari R, Naderi NJ, Razavi AE. A retrospective institutional study of histopathologic pattern of Oral Squamous Cell Carcinoma (OSCC) in Tehran, Iran during 2006-2015. J Res Med Sci $2019 ; 24$. 
2. Pires FR, Ramos AB, Oliveira JBC de, Tavares AS, Luz PSR da, Santos TCRB dos. Oral squamous cell carcinoma: clinicopathological features from 346 cases from a single oral pathology service during an 8-year period. J Appl Oral Sci 2013;21(5):460-7.

3. Markopoulos AK. Current aspects of oral squamous cell carcinoma. Open Dent J 2012;6:126.

4. Sridharan G, Ramani P, Patankar S, Vijayaraghavan R. Evaluation of salivary metabolomics in oral leukoplakia and oral squamous cell carcinoma. J Oral Pathol Med 2019;48(4):299-306.

5. Sridharan G, Ramani P, Patankar S. Serum metabolomics in oral leukoplakia and oral squamous cell carcinoma. J Cancer Res Ther 2017;13(3):556-61.

6. Thangaraj SV, Shyamsundar V, Krishnamurthy A, Ramani P, Ganesan K, Muthuswami M, et al. Molecular Portrait of Oral Tongue Squamous Cell Carcinoma Shown by Integrative Meta-Analysis of Expression Profiles with Validations. Plos One 2016;11:e0156582.

7. Premkumar J, Ramani P, Chandrasekar T, Natesan A, Premkumar P. Detection of species diversity in oral candida colonization and anti-fungal susceptibility among non-oral habit adult diabetic patients. J Nat Sci Biol Med 2014;5(1):148-54.

8. Ranganathan K, Rooban T, Rao UM. Oral squamous cell carcinoma in patients with and without predisposing habits in glossal and extra-glossal site: An institutional experience in South India. Indian J Cancer 2015;52(4):625-7.

9. Salian V, Dinakar C, Shetty P, Ajila V. Etiological Trends in Oral Squamous Cell Carcinoma: A Retrospective Institutional Study. Cancer Transl Med 2016;2(2):33.

10. Tandon P, Dadhich A, Saluja H, Bawane S, Sachdeva S. The prevalence of squamous cell carcinoma in different sites of oral cavity at our Rural Health Care Centre in Loni, Maharashtra - a retrospective 10-year study. Contemp Oncol 2017;21(2):178-83.

11. Hannah R, Ramani P, Sherlin HJ, Ranjith G, Ramasubramanian A, Jayaraj G, et al. Awareness about the use, ethics and scope of dental photography among undergraduate dental students dentist behind the lens. Res J Pharm Tech 2018;11(3):1012-6.

12. Shree KH, Ramani P, Sherlin H, Sukumaran G, Jeyaraj G, Don $\mathrm{KR}$, et al. Saliva as a diagnostic tool in oral squamous cell carcinoma--a systematic review with Meta analysis. Pathol Oncol Res 2019;25(2):447-53.

13. Gupta V, Ramani P. Histologic and immunohistochemical evaluation of mirror image biopsies in oral squamous cell carcinoma. J Oral Bio Craniofac Res 2016;6(3):194-7.

14. Jayaraj G, Sherlin HJ, Ramani P, Premkumar P, Natesan A. Stromal myofibroblasts in oral squamous cell carcinoma and potentially malignant disorders. Indian J Cancer 2015;52(1):87-92.

15. Viveka TS, Shyamsundar V, Krishnamurthy A, Ramani P, Ramshankar V. p53 expression helps identify high risk oral tongue pre-malignant lesions and correlates with patterns of invasive tumour front and tumour depth in oral tongue squamous cell carcinoma cases. Asian Pac J Cancer Prev 2016;17(1):189-95.

16. Coelho KR. Challenges of the oral cancer burden in India. J Cancer Epidemiol 2012;2012:701932.

17. Kumar A, Sherlin HJ, Ramani P, Natesan A, Premkumar P, Others. Expression of CD 68, CD 45 and human leukocyte antigenDR in central and peripheral giant cell granuloma, giant cell tumor of long bones, and tuberculous granuloma: An immunohistochemical study. Indian J Dent Res 2015;26(3):295.

18. Shenoi R, Devrukhkar V, Chaudhuri, Sharma BK, Sapre SB, Chikhale A. Demographic and clinical profile of oral squamous cell carcinoma patients: a retrospective study. Indian J Cancer 2012;49(1):21-6.

19. Swathy S, Gheena S, Varsha SL. Prevalence of pulp stones in patients with a history of cardiac diseases. Res J Pharm Tech 2015;6:37-43.

20. Iype EM, Pandey M, Mathew A, Thomas G, Sebastian P, Nair MK. Oral cancer among patients under the age of 35 years. J Postgrad Med 2001;47(3):171-6.

21. Mesquita JA, Cavalvanti AL, Nonaka CFW, Godoy GP, Alves PM. Clinical and histopathological evidence of oral squamous cell carcinoma in young patients: systematized review. J Bras Patol Med Lab 2014;50(1):67-74.

22. Jangid K, Alexander AJ, Jayakumar ND, Varghese S, Ramani P. Ankyloglossia with cleft lip: A rare case report. J Indian Soc Periodontol 2015;19(6):690.

23. Sivaramakrishnan SM, Ramani P. Study on the Prevalence of Eruption Status of Third Molars in the South Indian Population. Early Pregnancy 2015;7(4):1.

24. Sharma P, Saxena S, Aggarwal P. Trends in the epidemiology of oral squamous cell carcinoma in Western UP: an institutional study. Indian J Dent Res 2010;21(3):316-9.

25. Alves AM, Diel LF, Lamers ML. Macrophages and prognosis of oral squamous cell carcinoma: A systematic review. J Oral Pathol Med 2018;47(5):460-7.

26. Pindborg JJ, Mehta FS, Gupta PC, Daftary DK, Smith CJ. Reverse smoking in Andhra Pradesh, India: a study of palatal lesions among 10,169 villagers. Br J Cancer 1971;25(1):10-20.

27. Kayal L, Jayachandran S, Bhaskar YH. Squamous Cell Carcinoma of Tongue-A Case Report And Review Of Literature. Int J Curr Res Rev 2016;3:34-40.

28. Mack TM. The new pan-Asian paan problem. Lancet 2001;357(9269):1638-9.

29. Merchant A, Husain SS, Hosain M, Fikree FF, Pitiphat W, Siddiqui $\mathrm{AR}$, et al. Paan without tobacco: an independent risk factor for oral cancer. Int J Cancer 2000;86(1):128-31.

30. Rao SVK, Mejia G, Roberts-Thomson K, Logan R. Epidemiology of Oral Cancer in Asia in the Past Decade- An Update (2000-2012). Asian Pac J Cancer Prev 2013;14:5567-77.

31. Fang KH, Kao HK, Cheng MH, Chang YL, Tsang NM, Huang $\mathrm{YC}$, et al. Histological differentiation of primary oral squamous cell carcinomas in an area of betel quid chewing prevalence. Otolaryngol Head Neck Surg 2009;141(6):743-9.

32. Jayaraj G, Sherlin HJ, Ramani P, Premkumar P, Anuja N. Cytomegalovirus and Mucoepidermoid carcinoma: A possible causal relationship? A pilot study. J Oral Maxillofac Pathol 2015;19(3):319.

33. Jayaraj G, Ramani P, Herald J. Sherlin, Premkumar P, Anuja N. Inter-observer agreement in grading oral epithelial dysplasia - A systematic review. J Oral Maxillofac Pathol 2015;27:112-6.

34. Gheena S, Ezhilarasan D. Syringic acid triggers reactive oxygen species-mediated cytotoxicity in HepG2 cells. Hum Exp Toxicol 2019;38(6):694-702. .

35. Ramasubramanian A, Ramani P, Sherlin HJ, Premkumar P, Natesan A, Thiruvengadam C. Immunohistochemical evaluation of oral epithelial dysplasia using cyclin-D1, p27 and p63 expression as predictors of malignant transformation. J Nat Sci Biol Med. 2013;4(2):349-358. 